\title{
Effects of Continuous Nursing Intervention on Postoperative Rehabilitation of Patients with Craniocerebral Trauma
}

\author{
Xiangyun Rong* \\ Changzhou Medical District, 904 Hospital of the Joint Logistics Support Force of the Chinese People's Liberation Army, \\ Changzhou 213000, Jiangsu Province, China
}

*Corresponding author: Xiangyun Rong, 747298700@qq.com

Copyright: (C) 2022 Author(s). This is an open-access article distributed under the terms of the Creative Commons Attribution License (CC BY 4.0), permitting distribution and reproduction in any medium, provided the original work is cited.

\begin{abstract}
Objective: To study and analyze the clinical effect of continuous nursing in the process of postoperative rehabilitation for patients with craniocerebral trauma. Methods: From August 2018 to July 2021, 40 patients who came to our hospital for craniocerebral trauma treatment were randomly selected as clinical experimental research objects, and they were divided into continuous nursing group and routine nursing group. The rehabilitation of patients in the two groups before and after postoperative nursing was observed and counted. Results: Before nursing intervention, there was no significant difference in MMSE score and Fugl-Meyer score between the two groups, $p>0.05$. After nursing, the scores were scored again at 3 and 6 months after nursing. It was found that the two groups were improved, but the improvement range in the continuous nursing group was significantly higher than that in the routine nursing group, the difference was statistically significant $(p<0.05)$. From the score of quality of life, the scores of physical pain, psychological function, mental health and social function in the continuous nursing group were better than those in the routine nursing group, the difference was statistically significant $(p<0.05)$. Conclusion: Using continuous nursing measures for rehabilitation nursing of patients with craniocerebral trauma after discharge can significantly improve the quality of life of patients, restore the mental state and limb function of patients, and has significant clinical effect.
\end{abstract}

Keywords: Continuous nursing intervention; Craniocerebral trauma; Postoperative rehabilitation

Online publication: January 19, 2022

\section{Introduction}

Craniocerebral trauma is a common disease in neurosurgery, which has the characteristics of high disability rate and high mortality. In recent years, with the progress of medical science and technology and the improvement of medical level, great progress has been made in the emergency treatment of craniocerebral trauma. Most patients can be treated effectively to reduce the death of patients due to disease ${ }^{[1]}$. However, patients with craniocerebral trauma need to go through a long time in the rehabilitation stage after treatment, and because patients will have functional disorders in varying degrees, such as exercise, emotion, cognition and language, it will have a serious negative impact on their own quality of life and family. Therefore, we should not only pay attention to the first aid of craniocerebral trauma, but also give sufficient and effective nursing intervention measures to patients with craniocerebral trauma in the process of postoperative rehabilitation, so as to better promote the recovery of patients' body related functions and improve their quality of life. Continuous nursing is a means of continuous nursing for patients in different places through 
the formulation of nursing plan. Nursing guidance can be given no matter whether the patients are hospitalized, referred, discharged, returned to the family and other scenes ${ }^{[2]}$. Based on the above research, this paper starts from August 2018 and ends in July 2021. During this period, 40 patients who came to our hospital for craniocerebral trauma treatment were randomly selected as the clinical experimental research object to carry out the control experiment of continuous nursing, so as to understand the nursing effect.

\section{Materials and methods}

\subsection{General information}

From August 2018 to July 2021, 40 patients randomly selected for craniocerebral trauma treatment in our hospital during this period were selected as clinical experimental research objects, and they were divided into continuous care group and routine care group. In the routine nursing group, there were 12 males and 8 females. The maximum age of the patients was 76 years old and the minimum was 45 years old, with an average of $(60.08 \pm 5.21)$ years old. According to the type of brain injury, there were 3 cases of subdural hematoma, 2 cases of epidural hematoma, 8 cases of brain stem injury, 2 cases of cerebral contusion and laceration, and 5 cases of scalp contusion and laceration. From the pathogenic causes, there were 6 patients with car accidents, 5 patients with high-altitude falling and 9 patients with falling injuries. In the continuous care group, there were 11 males and 9 females. The maximum age of the patients was 75 years old and the minimum was 45 years old, with an average of $(60.43 \pm 4.98)$ years old. According to the type of brain injury, there were 4 cases of subdural hematoma, 3 cases of epidural hematoma, 7 cases of brain stem injury, 2 cases of cerebral contusion and laceration, and 4 cases of scalp contusion and laceration; From the pathogenic causes, there were 7 patients with car accidents, 5 patients with high-altitude falling and 8 patients with falling injuries.

All patients in this experiment know the purpose and content of this experiment and voluntarily participate in this experiment. The experiment was approved by the ethics committee of our hospital before implementation. The above two groups were comparable in general data, $\mathrm{P}>0.05$.

\subsubsection{Inclusion criteria}

(1) All patients were diagnosed as craniocerebral trauma.

(2) All patients were adults and under 80 years old.

(3) After treatment, the patient's emotional state was stable and could be discharged.

\subsubsection{Exclusion criteria}

(1) The patient had coma, unconsciousness and mental disorder.

(2) Patients had other organ diseases.

(3) Patients with poor compliance.

\subsection{Methods}

For the patients in the routine nursing group, routine nursing was carried out. The nursing content was to introduce the discharge related matters to the patients, distribute the out of hospital rehabilitation manual and provide out of hospital rehabilitation guidance. A telephone follow-up was conducted one week after discharge, asking about medication and diet, recording the condition and understanding rehabilitation training. Telephone follow-up will be carried out irregularly in the future ${ }^{[3]}$.

For the patients in the continuous care group, the continuous care method was adopted, and the nursing contents included:

(1) A continuing nursing team was set up, including a head nurse, a ward assistant and a responsible nurse. The head nurse acted as the team leader. According to the principle of responsibility management, the 
head nurse supervised the specific implementation of continuous nursing, regularly carried out group meetings, analyzed the problems faced, so as to find countermeasures for continuous improvement and continuously improve nursing quality. The main responsibility of ward assistant was to establish management files for discharged patients with craniocerebral trauma, including general information such as age and gender, as well as medical information such as length of stay and condition. The responsible nurse was responsible for building a WeChat group, which was established before the patient was discharged from the hospital. The members included the responsible nurse, ward assistant, patient and family members. In the later stage, the patients would receive rehabilitation guidance through the communication platform of WeChat Group ${ }^{[4]}$.

(2) Patients were systematically followed up by telephone after discharge, mainly by ward assistants. They were called once a week within one month after discharge and followed up once in the second half of the month. Telephone follow-up was conducted once a month for 4 months after discharge. The patients were followed up for half a year.

(3) Home care out of hospital. The responsible nurse uploaded video data of postoperative rehabilitation of various craniocerebral trauma to the WeChat group. The video content included body movement, daily life, language ability, attention and other training. The patient's family members were required to give help and support as well as cooperate with the patient to complete the video content. If necessary, they could contact the ward assistant for on-site guidance. It is necessary to ensure that patients and family members correctly master the content.

(4) In order to facilitate communication and interaction, and taking into account the daily work of nurses, it is agreed that at 7 p.m. every day, patients and their families can take the initiative to ask questions in the WeChat group, and the nurses will answer them ${ }^{[5]}$.

\subsection{Statistical treatments}

In this study, the relevant data were processed by statistical software SPSS 21.0.

\section{Results}

3.1. Comparison of MMSE and Fugl-Meyer scores between the two groups before and after nursing Before nursing intervention, there was no significant difference in MMSE score and Fugl-Meyer score between the two groups, $p>0.05$. After nursing, the scores were scored again at 3 and 6 months after nursing. It was found that the two groups were improved, but the improvement range in the continuous nursing group was significantly higher than that in the routine nursing group, the difference was statistically significant $(\mathrm{p}<0.05)$. (Details can be seen in Table $\mathbf{1})$.

Table 1. Comparison of MMSE and Fugl-Meyer scores between the two groups before and after nursing

\begin{tabular}{|c|c|c|c|c|c|c|}
\hline \multirow[t]{2}{*}{ Group } & \multicolumn{3}{|c|}{ MMSE score } & \multicolumn{3}{|c|}{ Fugl Meyer score } \\
\hline & $\begin{array}{c}\text { Before } \\
\text { nursing }\end{array}$ & $\begin{array}{c}3 \text { months after } \\
\text { nursing }\end{array}$ & $\begin{array}{c}6 \text { months after } \\
\text { nursing }\end{array}$ & $\begin{array}{c}\text { Before } \\
\text { nursing }\end{array}$ & $\begin{array}{c}3 \text { months after } \\
\text { nursing }\end{array}$ & $\begin{array}{c}6 \text { months after } \\
\text { nursing }\end{array}$ \\
\hline $\begin{array}{l}\text { Continuous } \\
\text { nursing group }\end{array}$ & $14.62 \pm 3.55$ & $20.33 \pm 4.21$ & $29.65 \pm 5.09$ & $30.45 \pm 3.44$ & $46.98 \pm 6.14$ & $55.27 \pm 6.56$ \\
\hline $\begin{array}{l}\text { Routine nursing } \\
\text { group }\end{array}$ & $14.77 \pm 3.92$ & $17.56 \pm 4.35$ & $25.44 \pm 4.97$ & $30.38 \pm 3.57$ & $35.17 \pm 4.52$ & $42.62 \pm 5.84$ \\
\hline t value & 0.213 & 3.246 & 4.187 & 0.129 & 10.948 & 10.189 \\
\hline $\mathrm{p}$ value & $>0.05$ & $<0.05$ & $<0.05$ & $>0.05$ & $<0.05$ & $<0.05$ \\
\hline
\end{tabular}




\subsection{Comparison of quality-of-life score between the two groups before and after nursing}

From the score of quality of life, the scores of physical pain, psychological function, mental health and social function in the continuous nursing group were better than those in the routine nursing group, the difference was statistically significant $(\mathrm{p}<0.05)$. (Details can be seen in Table 2).

Table 2. Comparison of quality-of-life score between the two groups before and after nursing

\begin{tabular}{ccccc}
\hline Group & Physical pain & Psychological function & Mental health & Social function \\
\hline Continuous nursing group & $50.46 \pm 7.64$ & $55.54 \pm 6.27$ & $58.54 \pm 8.35$ & $60.57 \pm 5.47$ \\
Routine nursing group & $39.61 \pm 7.54$ & $42.65 \pm 6.53$ & $45.33 \pm 8.66$ & $49.28 \pm 5.64$ \\
t value & 7.138 & 10.087 & 7.783 & 10.183 \\
p value & $<0.05$ & $<0.05$ & $<0.05$ & $<0.05$ \\
\hline
\end{tabular}

\section{Discussion}

Craniocerebral trauma is the injury of the head caused by external violence. The clinical treatment is generally surgical, and it needs a long time to recover after operation. Rehabilitation is mostly carried out in families outside the hospital. Due to the lack of professional rehabilitation training knowledge of patients and their families, it will have an impact on rehabilitation ${ }^{[6]}$. In order to achieve the unity of economy and effect, it is necessary to extend nursing measures to family rehabilitation. Continuous nursing intervention is a scientific and systematic family rehabilitation nursing measure, which can meet the needs of patients and their families as well as get professional nursing and rehabilitation guidance at home ${ }^{[7]}$. In the research of this paper, before nursing intervention, there was no significant difference in MMSE score and FuglMeyer score between the two groups, $\mathrm{p}>0.05$. After nursing intervention, the scores were scored again at 3 and 6 months after nursing. It was found that the two groups were improved, but the improvement range in the continuous nursing group was significantly higher than that in the routine nursing group, the difference was statistically significant $(\mathrm{p}<0.05)$. From the score of quality of life, the scores of physical pain, psychological function, mental health and social function in the continuous nursing group were better than those in the routine nursing group, the difference was statistically significant $(\mathrm{p}<0.05)$.

To summarize, utilizing continuous nursing measures for post-discharge rehabilitation nursing of patients with craniocerebral trauma can enhance patients' quality of life, recover mental state, and limb function, and has a significant clinical effect.

\section{Disclosure statement}

The author declares no conflict of interest.

\section{References}

[1] Li D, Liu X, 2019, Effect of Staged Rehabilitation Nursing Mode on Postoperative Rehabilitation Effect of Patients with Craniocerebral Trauma. Henan Medical Research, 28(4): 742-743.

[2] Feng F, 2021, Application Effect of Continuous Nursing in Patients with Craniocerebral Trauma in Postoperative Recovery Period. Practical Clinical Journal of Integrated Traditional Chinese and Western Medicine, 21(11): 2.

[3] $\mathrm{Hu}$ X, 2016, Influence of Staged Rehabilitation Nursing on Limb Movement and Nerve Function of Patients with Craniocerebral Trauma after Surgery. Journal of Clinical Medicine in Practice, 20(8): 77$79,86$.

[4] Deng N, 2019, Analysis of Nursing Effect of Continuous Nursing Intervention on Patients with 
Craniocerebral Trauma. Journal of Frontier of Medicine, 9(30): 170.

[5] Shi H, 2017, Clinical Effect Evaluation of Continuous Nursing in Patients with Craniocerebral Trauma After Discharge. Today Nurse: Academic Edition (Mid-term Issue), 10(5): 40-41.

[6] Meng Z, 2021, Effect of Comprehensive Rehabilitation Nursing on Postoperative Curative Effect of Patients with Craniocerebral Trauma. Chinese Medical Journal of Metallurgical Industry, 38(2): 229230.

[7] Wang Q, Tian Q, 2021, Effect of Continuous Nursing on Postoperative Rehabilitation of Patients with Craniocerebral Trauma. Clinical Research and Practice, 6(1): 151-152.

Publisher's note

Bio-Byword Scientific Publishing remains neutral with regard to jurisdictional claims in published maps and institutional affiliations. 